\title{
Electromagnetic Study of the Chlorosome Antenna Complex of Chlorobium tepidum
}

\section{Citation}

Valleau, Stéphanie, Semion K. Saikin, Davood Ansari-Oghol-Beig, Masoud Rostami, Hossein Mossallaei, and Alán Aspuru-Guzik. 2014. "Electromagnetic Study of the Chlorosome Antenna Complex of Chlorobium Tepidum." ACS Nano 8 (4) (April 22): 3884-3894. doi:10.1021/nn500759k.

\section{Published Version}

doi: $10.1021 / \mathrm{nn} 500759 \mathrm{k}$

\section{Permanent link}

http://nrs.harvard.edu/urn-3:HUL.InstRepos:23603186

\section{Terms of Use}

This article was downloaded from Harvard University's DASH repository, and is made available under the terms and conditions applicable to Open Access Policy Articles, as set forth at http:// nrs.harvard.edu/urn-3:HUL.InstRepos:dash.current.terms-of-use\#OAP

\section{Share Your Story}

The Harvard community has made this article openly available.

Please share how this access benefits you. Submit a story.

Accessibility 


\title{
Electromagnetic study of the chlorosome antenna complex of Chlorobium-tepidum
}

\author{
Stéphanie Valleau, ${ }^{*},{ }^{\dagger}$ Semion K. Saikin, ${ }^{\dagger}$ Davood Ansari-Oghol-Beig, ${ }^{\dagger}$ \\ Masoud Rostami, ${ }^{\ddagger}$ Hossein Mossallaei, ${ }^{\ddagger}$ and Alán Aspuru-Guzik ${ }^{*, \dagger}$ \\ Department of Chemistry and Chemical Biology, Harvard University, Cambridge, Massachusetts \\ 02138, USA, and CEM and Photonics Lab, Electrical and computer engineering department, \\ Northeastern University, Boston, Massachusetts, USA \\ E-mail: svalleau@fas.harvard.edu; aspuru@chemistry.harvard.edu
}

\begin{abstract}
Green sulfur bacteria is an iconic example of nature's adaptation: thriving in environments of extremely low photon density, the bacterium ranks itself amongst the most efficient natural lightharvesting organisms. The photosynthetic antenna complex of this bacterium is a self-assembled nanostructure, $\approx 60 \times 150 \mathrm{~nm}$, made of bacteriochlorophyll molecules. We study the system from a computational nanoscience perspective by using electrodynamic modeling with the goal of understanding its role as a nanoantenna. Three different nanostructures, built from two molecular packing moieties, are considered: a structure built of concentric cylinders of aggregated bacteriochlorophyll-d monomers, a single cylinder of bacteriochlorophyll-c monomers and a model for the entire chlorosome. The theoretical model captures both coherent and incoherent components of exciton transfer. The model is employed to extract optical spectra, concentration and depolarization of electromagnetic fields within the chlorosome, and fluxes of energy transfer for the structures. The second model nanostructure shows the largest field enhancement. Further, field enhancement is found to be more sensitive to dynamic noise rather than structural disorder. Field depolarization however is similar for all structures. This
\end{abstract}

\footnotetext{
*To whom correspondence should be addressed

${ }^{\dagger}$ Harvard University

${ }^{\ddagger}$ Northeastern University
}

indicates that the directionality of transfer is robust to structural variations while on the other hand, the intensity of transfer can be tuned by structural variations.

keywords: electrodynamics, chlorosome, antenna, field enhancement, spectra, classical

$\mathrm{T}$ HE life cycle of plants, photosynthetic bacteria and algae is based on the harvesting of solar energy. In all of these organisms solar light is absorbed and processed by a photosynthetic system. This unit typically consists of an aggregate of light absorbing molecules, e.g. bacteriochlorophylls (BChls) (See Fig. 1, panel II). Photosynthetic systems vary in composition and size, for instance, their dimensions can range from tens to hundreds of nanometers with up to $\sim 2 \cdot 10^{5}$ pigment molecules. Solar energy is transferred in these systems through molecular excitations known as excitons. Success in nature's competition for resources is crucial for the survival of phototrophic organisms. Therefore, optimal efficiency of light absorption and energy transfer within the photosynthetic systems are essential characteristics.

Recently, much scientific effort has been de- 
I) NATURAL SYSTEM

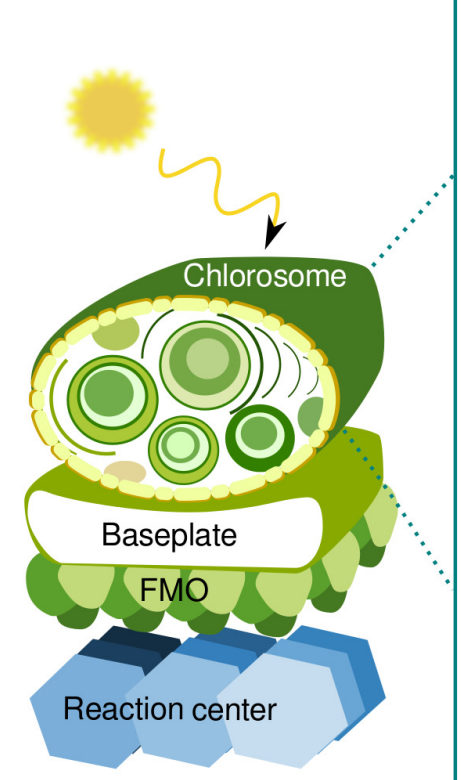

II) ATOMISTIC MODEL FOR THE CHLOROSOME

a)

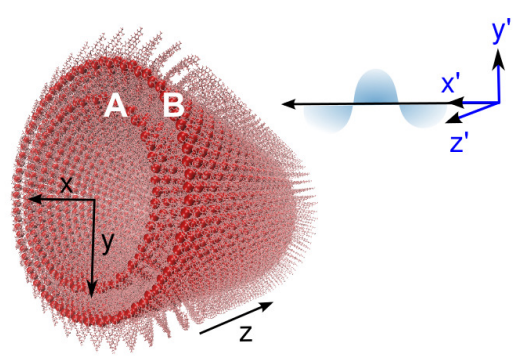

Roll structure - mutant

b)

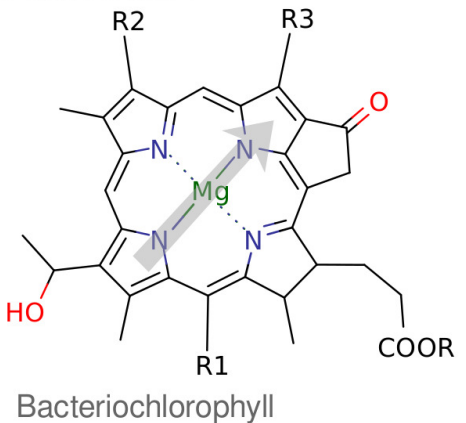

III) CLASSICAL DIPOLE MODELS - DIPOLE PACKING

a)

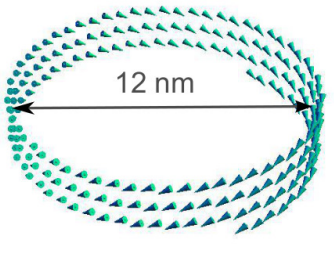

Roll A - mutant b)

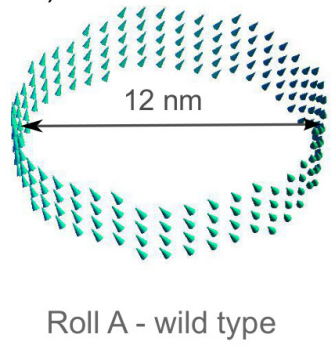

c)

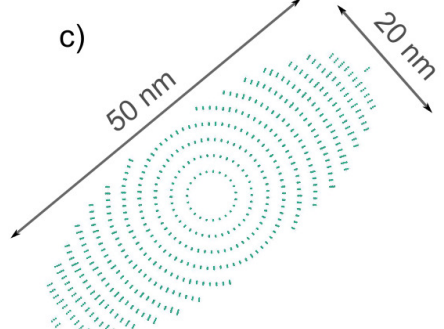

Chlorosome - wild type

Figure 1: Panel I) - model of the photosynthetic units in the natural system of green sulfur bacteria the main elements are the chlorosome, the baseplate and the FMO complexes which transfer excitons to the reaction center. Panel II): Atomistic model for the chlorosome (Ref. 2); in a) the chlorosome nanostructure consisting of two concentric rolls, roll A and B, is shown. Further, the reference system for the incoming field propagating along the $x^{\prime}$ direction is indicated. In $\mathrm{b}$ ) the molecular structure of a bacteriochlorophyll (BChl), the main building block of chlorosomes, is indicated. Here, the R, R1, R2 and R3 symbols represent molecular chains (not drawn for simplicity) which vary depending on the type of BChl. The grey arrow indicates the direction of the transition dipole for the first molecular optical transition. Panel III): three classical dipole models for the chlorosome nanostructure. In a) we see a slice of a single cylinder (full length $\sim 30 \mathrm{~nm}$ ) and the molecular packing of BChl-d as obtained by Ganapathy, et al. (Ref. 2). In b) another type of packing using BChl-c molecules is shown (full length 30nm). Finally in c) we show a slice of the model for the entire chlorosome nanostructure. This last structure is built using the packing motif of panel b). Due to the type of pigment molecules, structures b) and c) are denoted as "wild-type" structures. See Methods and Supporting Information for more details.

voted to understanding the microscopic principles which govern the efficiency of photosynthetic systems. ${ }^{1}$ Amongst these systems, green sulfur bacteria is one of the most widely studied. The photosynthetic system of green sulfur bacteria consists of three main elements (See Fig. 1, panel I). The first is the chlorosome: a large nanostructure array of BChl's which functions as a light absorbing antennae. The second elements are intermediates (these include the baseplate and the FennaMatthews-Olson protein complex) which play the role of exciton bridges connecting the chlorosome to the third and last element, the reaction center, where the exciton energy is employed in the synthesis of metabolic compounds.

Similarly, in the nanostructure community, much research has focused on the study of nanoantennas. ${ }^{3-6}$ Various types of antennas have been devised and studied ${ }^{7,8}$ in order to understand which materials and shapes are optimal to enhance and direct radiation. Models for nonradiative energy transfer between nanostructures have also been developed ${ }^{9}$ in an effort to answer these questions. 
It is interesting that nature has evolved to generate antennas in biological organisms as well. The chlorosome nanostructure has dimensions of the order of hundreds of nanometers and works exactly like an antenna: it absorbs photons and transmits them to the next subunits very efficiently. A question naturally arises: can energy transfer principles be deduced from the study of these natural systems and applied to the field of nano-antennas? To begin to answer this question, in this paper, we investigate the electromagnetic properties of the chlorosome antenna complex.

Previously, we investigated reduced models of this photosynthetic unit using open-quantum system approaches coupled to ab-initio simulations. Energy transfer was found to be a non-Markovian process ${ }^{10}$ which can be characterized by multiple timescales. ${ }^{11}$ Recently, some of us investigated a reduced model of the entire photosynthetic system atomistically, Ref. 12. We found energy transfer to be robust to initial conditions and temperature. Other theoretical studies on energy transfer dynamics as well as the spectroscopy of the antenna complex have been carried out for small models of the chlorosome structure ${ }^{13}$ using open-quantum system theories. Spectra were also obtained for helical aggregates using the CES approximation and including the vibrational structure. ${ }^{14}$ In this paper, we present a new perspective: an electrodynamic study of the full chlorosome antenna.

The chlorosome antenna complex is composed of up to tens of thousands of BChls (see Fig. 1) which makes it the largest of the photosynthetic antenna units known. This nanostructure is thought to be the main element responsible for capturing photons at the extremely low photon densities of the bacteria's environment. ${ }^{15,16}$ Quantum mechanical models, as employed in some of the recent theoretical work on photosynthetic systems, ${ }^{10,11,17-21}$ cannot be used here due to the large size of the full chlorosome antenna complex. Electrodynamic modeling thus provides a viable alternative. This approach can capture, within certain approximations, both coherent and incoherent excitation dynamics and has already been described and employed in the simple case of a molecular dimer. ${ }^{22}$ Further, we have recently ${ }^{23}$ devised an algorithm to solve the electrodynamic equations very efficiently even in the presence of thousands of molecules.

The chlorosome antenna complexes are composed of different types of BChls, namely BChlc, BChl-d or BChl-e, with varying chemical composition according to the species in question. Because of the large amount of disorder present in the natural system, the definitive structure for the complex is unknown. However, several models have been proposed. ${ }^{2,24-27}$ Recently, Ganapathy et al. ${ }^{2}$ determined the structure of a synthetic triple mutant chlorosome antennae, generated to mimic natural chlorosomes. This mutant structure replicated various structural signatures of the natural chlorosome while, however, being less efficient in terms of growth rates at different light intensities respect to the wild type chlorosome. Other experimental efforts have been made in this direction using a combination of NMR and X-Ray diffraction. ${ }^{28-30}$ The structure by Ganapathy et al. will be the first of three nanostructures which we will consider in this work and their experimental findings inspire the remaining two structures. The first structure comprises a series of concentric cylinders of aggregated BChl-d molecules (Fig. 1 II-a and III-a). We will consider both the case of a single cylinder and of two concentric cylinders in this work. The second system is a similar cylindrical array, built of BChl-c molecules rather than BChl-d (See Fig. 1 III-b). This structure is obtained following the findings of Ganapathy et al. ${ }^{2}$ Finally we will consider a model for the entire chlorosome (panel III-c)) consisting of over 70000 BChl-c. More detail on these three structures can be found in the Methods section.

Using our recent algorithm to solve the electromagnetic equations, ${ }^{23}$ we efficiently compute the induced polarization and fields of the three chlorosome structures. The role of different initial excitations, i.e frequency and polarization of the incoming field, are investigated. We also study the field enhancement and field depolarization as a function of structural disorder and dynamical noise. Finally, we determine fluxes of energy transferred in time to acceptors located around the antenna nanostructures. 


\section{Results and discussion}

\section{Antenna spectra and resonances}

The chlorosome antennae absorbs incoming light in the visible range. The resulting spectrum shows resonances determined by the presence of molecular transitions at about $750 \mathrm{~nm}$. The chlorosome aggregate resonances are shifted respect to the pigment transitions due to the couplings between monomers. In order to understand which regions are of interest for energy transfer, we calculated the absorption and circular dichroism spectra of the structures. Due to the three-dimensional arrangement of the dipoles in a cylindrical structure, we expect there to be two components in the absorption spectra, a $z$ polarization component, parallel to the main axis of the cylinder, and an in-plane $x y$ component, orthogonal to the main axis. Panel a) of Fig. 2 shows the computed absorption spectra for the roll structure III-a) of Fig. 1. The spectra were obtained using a semiclassical approach (see Methods) and using the Fermi-Golden rule quantum approach. The spectra were broadened by adding disorder through the rate $\Gamma=1 \mathrm{meV}$ (See Eq. 1). As remarked previously in the literature (see e.g. Ref. 31) the quantum and classical spectra are simply shifted (see Methods for more details). More importantly, in both approaches we see the expected $z$ and $x y$, components at about $\Delta \omega_{z}=-280 \mathrm{meV}$ and $\Delta \omega_{x y}=[-260 ;-230] \mathrm{meV}$ for the classical spectrum. A higher oscillator strength is observed for the $z$ component. The range over which transitions are observed is consistent with experiments ${ }^{32}$ though a direct comparison of the components is not possible due to the large amount of noise present in the natural system. The CD spectra in panel b) also follows the same trend ${ }^{1}$ as the experimentally observed CD, Ref. 32, 33. The alternating negative and positive peaks in the $\mathrm{CD}$ spectra are related to the orientation of the incoming field respect to the helicity of the structure. These spectra were also calculated for structure III-b) of Fig. 1 and these follow a similar pattern.

The inter- and intra-molecular vibrations, interaction with the solvent, and other environmen-

\footnotetext{
${ }^{1}$ Note that the structure of Ref. 2 was chosen with opposite helicity in agreement with experimental findings.
}

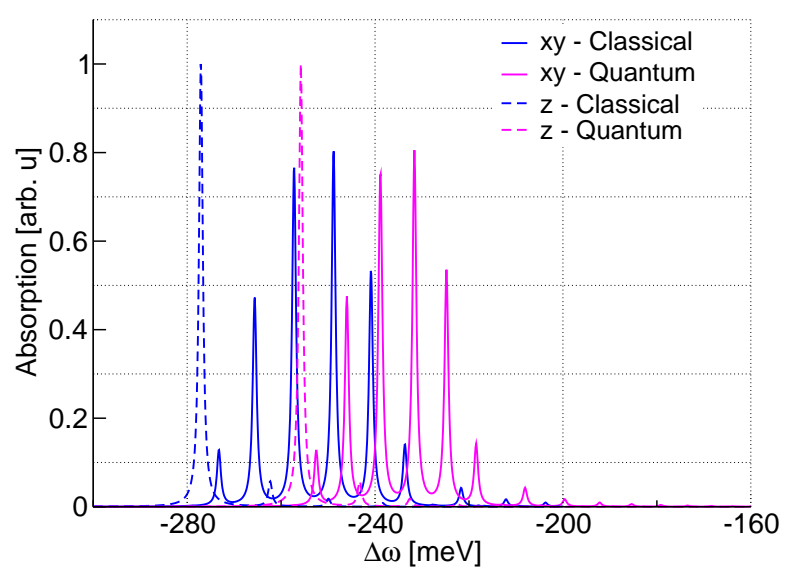

a)

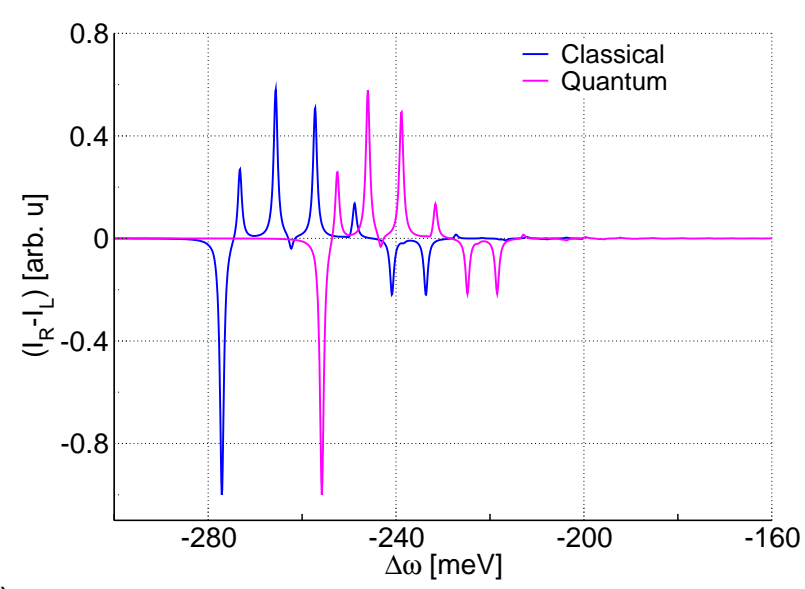

b)

Figure 2: Panel a) Absorption spectrum and its components obtained using a classical electrodynamic approach and the quantum Fermi-Golden rule approach. Panel b) Circular dichroism spectrum (CD) obtained using the classical and quantum approaches. The spectra were broadened with disorder $\Gamma=1 \mathrm{meV}$, and the frequency axis corresponds to the shift in energy respect to the monomer transition frequency. All spectra were computed for structure III-a) in Fig. 1. 
tal fluctuations, generate a source of noise which needs to be taken into account when modeling the system. Generally speaking, we can distinguish between static/structural disorder and dynamic noise based on the time scales of the associated fluctuations. Fluctuations related to structural disorder oscillate on a much longer timescale compared to the dynamics of the system whereas dynamical noise fluctuations are more rapid. The noise source is the same for both types of disorder.

In this model, one can include noise by introducing a molecular response function ${ }^{34}$

$$
\chi_{i, s}(\omega)=\frac{2}{\hbar} \frac{\left|\vec{\mu}_{i, s}\right|^{2} \omega_{i, s}}{\left(\omega_{i, s}+i \frac{\Gamma}{2}\right)^{2}-\omega^{2}} .
$$

Here, $\vec{\mu}_{i, s}$ is the transition dipole of the $s-t h$ transition for the $i-t h$ molecule and $\omega_{i, s}$ the corresponding transition frequency. The dynamical noise is accounted for by the rate constant, $\Gamma$, and structural disorder can be included by introducing noise in the transition frequencies, $\omega_{i, s}$. Usually, $\omega_{i, s}$, is taken from a Gaussian distribution, and structural disorder is characterized by the width of the Gaussian, $\sigma$.

There is no straightforward way to quantify structural and dynamical noise in the localized molecular basis experimentally. The sum of structural disorder, $\sigma$, and dynamic disorder, $\Gamma$, in the exciton, delocalized energy basis ${ }^{2}$ corresponds to the linewidth of the absorption spectrum. The overall disorder in the localized site basis should be of the same order of magnitude. In this case the linewidth is $\approx 70 \mathrm{meV}$ both for the BChlc monomeric spectrum and for the Chlorosome spectrum. ${ }^{35}$

\section{Induced fields}

Within the electromagnetic framework, we can obtain information on the exciton transfer properties from the induced polarizations. Indeed, once the nanostructure interacts with an incoming plane wave, the induced polarizations generate fields and thus transport can be quantified in terms of field enhancement. The directionality of transport can

\footnotetext{
${ }^{2}$ The exciton basis corresponds to the basis of delocalized energy states which is obtained by diagonalizing the Hamiltonian in the localized molecular basis.
}

also be determined by computing the field depolarization.

\section{Field concentration and depolarization}

Plane wave incoming fields propagating along the $x$ direction (see Fig. 1 panel II-a), were used to study the electric field enhancement and depolarization from the dipole arrays of the chlorosome. Two different polarizations of the incoming field are considered, along the $y^{\prime}$ direction and along the $z^{\prime}$ direction, as shown in Fig. 1, panel II-a.

The roll-A structure (Fig. 1; III-a), was employed to obtain the field enhancement $\kappa=\frac{|\vec{E}|}{\left|\vec{E}_{0}\right|}$ plots shown in Fig. 3. In these plots, we see the values of the scattered field calculated on a grid orthogonal to the structure (panels $a$ and $b$ ) and on a grid parallel to the structure (panels $\mathrm{c}$ and d). By comparison of panels a) and b) we notice a larger field enhancement when the incoming field is polarized along the $z^{\prime}$ direction. This is expected due to the more favorable overlap with the dipole orientations in the structure. The field enhancement overall is not very big in this case due to the large value of the noise $\Gamma=50 \mathrm{meV}$. We notice that the field is enhanced homogeneously in the radial direction (panels $\mathrm{b}, \mathrm{d}$ ), for $y^{\prime}$-polarized incoming field. This trend supports the idea of exciton transfer in the radial direction. This feature is observed even when including structural disorder. For the $z^{\prime}$-polarized incoming field (panels a,c), we see enhancement radially but also at the edges of the structure (panel c) this suggests that excitons may be transfered between layers and at the edges amongst neighboring substructures. In the next section, we look at how $\kappa$ varies with noise and structural disorder.

In Fig. 4, we show contour plots of the depolarization $\eta=\left(|\vec{E}|_{\|}-|\vec{E}|_{\perp}\right) /|\vec{E}|$ of the electric field on a horizontal grid across the structures. Panel a) and b) indicate $\eta$ computed for the roll A structure (Fig. 1; III-a) while panels c) and d) correspond to calculations for the chlorosome model structure with $7 \cdot 10^{4}$ molecules (Fig. 1; III-c).

In panels a) and c) the depolarization is calculated for a $z^{\prime}$-polarized incoming field and in panels b) and d) for a $y^{\prime}$-polarized incoming field. The depolarization, $\eta$, was also computed for the wild 


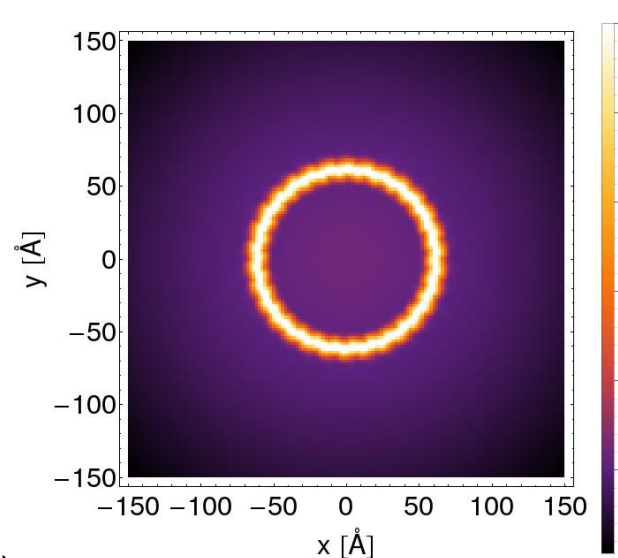

a)

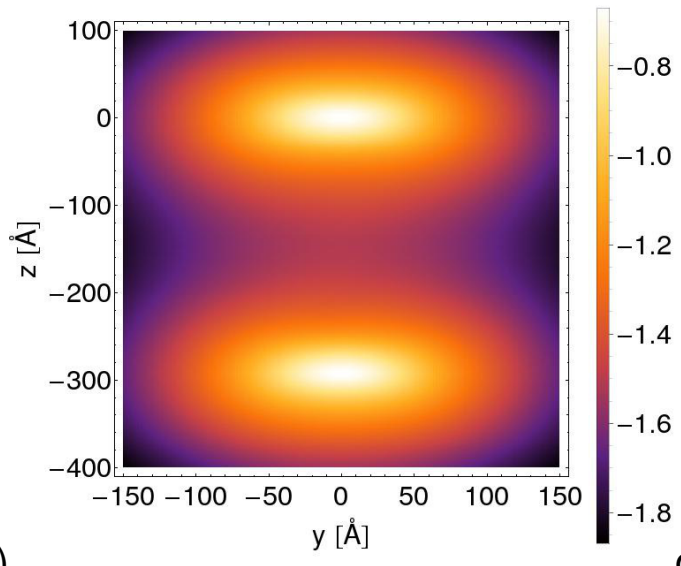

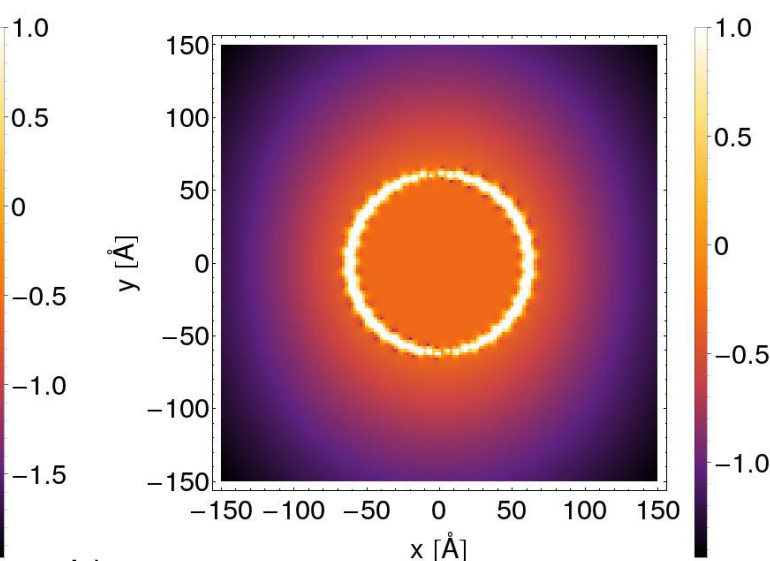

b)

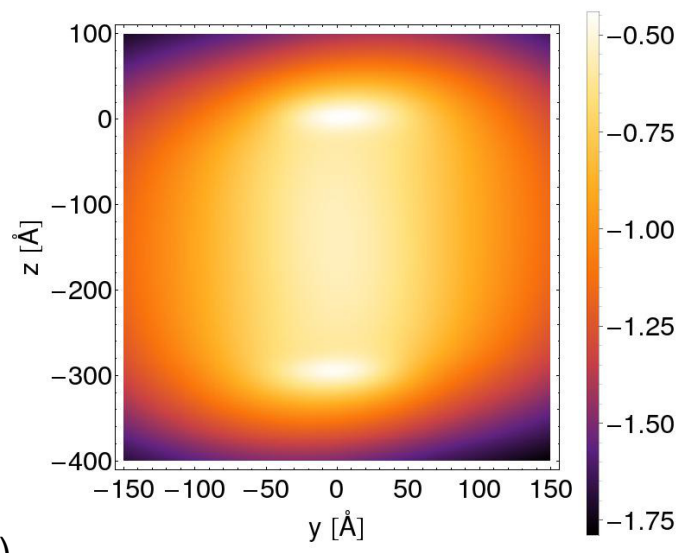

d)

Figure 3: Panel a) Base ten logarithm of the electric field enhancement, $\log _{10} \kappa$, calculated on a grid orthogonal to the roll structure in Fig. 1, III-a). The dynamical noise rate is $\Gamma=50 \mathrm{meV}$, the initial field excitation frequency is shifted by $\Delta \omega=0.23 \mathrm{eV}$ (see Fig. 2). The polarization of the initial field is along the $z^{\prime}$ direction. Panel b), same as panel a) but for external field polarized along the $y^{\prime}$ direction. Panel c) same as panel a) but here field is calculated on a grid parallel to the longest axis of the roll and at a distance of $r=80 \AA$ from the origin. Panel d) same as panel c) but for $y^{\prime}$-polarization. 


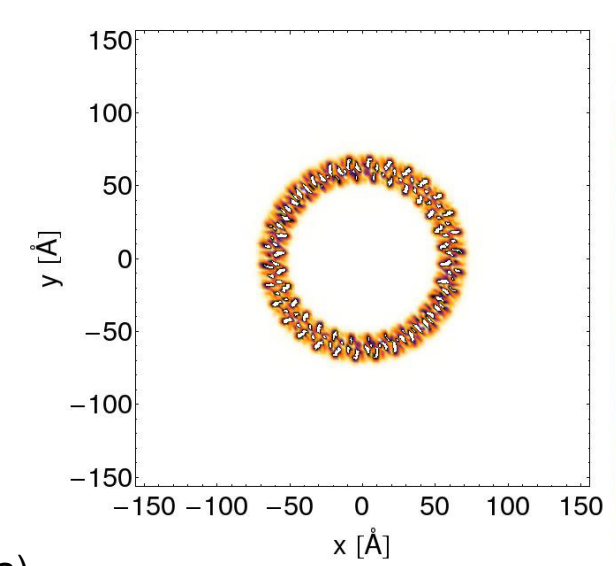

a)

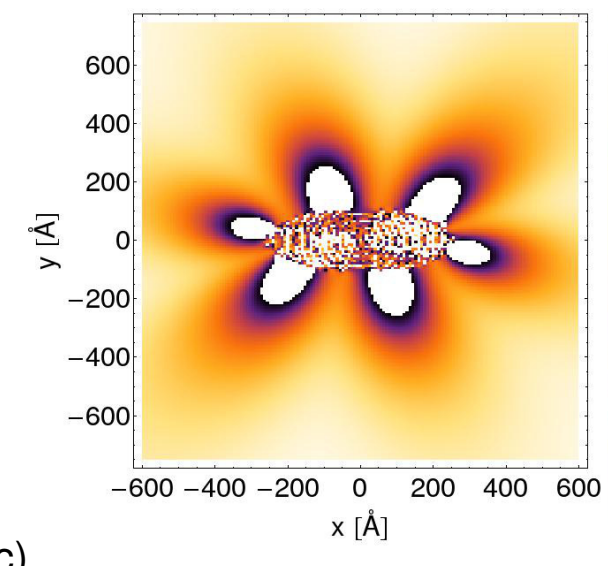

c)
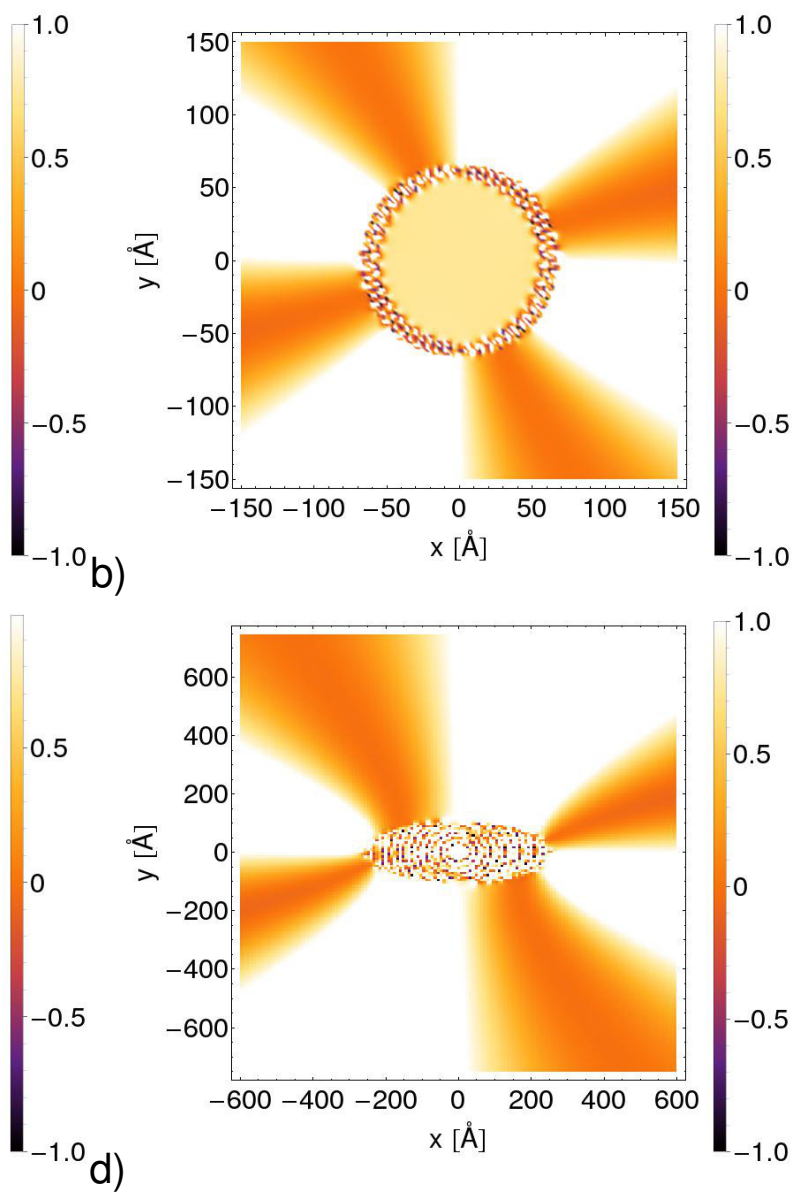

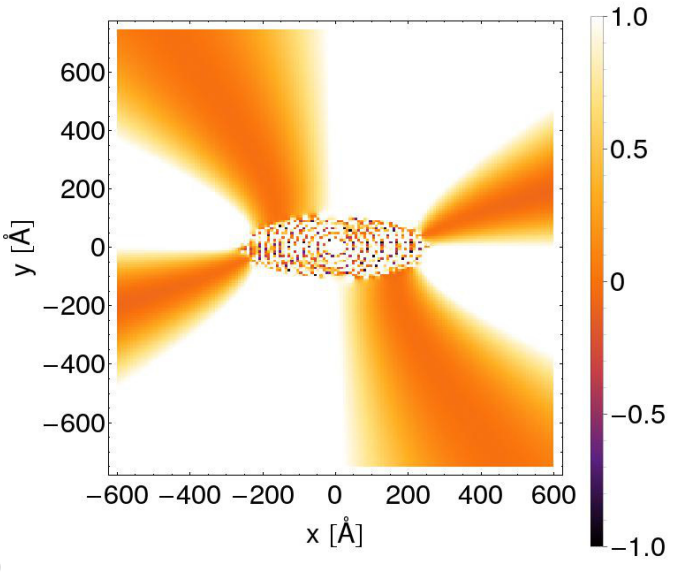

Figure 4: Panel a) Depolarization of electric field $\eta=\left(|\vec{E}|_{\|}-|\vec{E}|_{\perp}\right) /|\vec{E}|$ calculated on a grid orthogonal to the roll structure in Fig. 1, III-a). The dynamical noise rate is $\Gamma=50 \mathrm{meV}$, the initial field excitation frequency is shifted by $\Delta \omega=\omega_{0}-\omega_{\text {ext }}=0.23 \mathrm{eV}$ (See Fig. 2). The polarization of the initial field is along the $z^{\prime}$ direction. Panel b), same as panel a) but for $y^{\prime}$-polarization of the external field. Panels c) and d), same as a) and b) but for the wild type chlorosome model structure III-c) with $\Delta \omega=\omega_{0}-\omega_{\text {ext }}=0.22 \mathrm{eV}$. 
type roll-A structure (Fig. 1; III-b) and the pattern is analogous to that shown here in panels a) and b) for structure III-a. For these two cylindrical structures, the $z^{\prime}$-polarized incoming field is not significantly depolarized, this is probably due to the fact that the dipoles' $z$-component is largest.

For the third model chlorosome structure, we see in panel c) that the field gets depolarized in an interesting pattern which most likely originates from the dipole packing at the edges. For the $y^{\prime}$ polarized incoming field, the depolarization pattern is similar for all three structures (panels b, d) and the field remains polarized for specific directions, perhaps those corresponding to where other substructures might be found. The observed depolarization patterns ensure that a photon of arbitrary polarization will be transferred to the next layer following the radial direction.

The observed robustness to structural variations of the field polarization is in agreement with recent experimental findings by Tian et al., Ref. 36. In this work the authors used 2D fluorescence polarization microscopy on a series of wild type chlorosomes of C-tepidum grown in homogeneous conditions. They found that all spectral properties were homogeneous independent of the selected chlorosome and their results suggested that BChl molecules must possess a distinct organization within the chlorosomes. A similar organization is present in our model structures and it appears that even when adding structural disorder, the overall transition dipole moment components are not significantly modified and the largest component remains along the $z$ axis thus leading to the field polarization patterns we have obtained. It would be interesting to obtain a theoretical estimate of the modulation depth for each of the three structures so as to compare to these experimental findings.

\section{Scaling of field enhancement with disorder}

In Fig. 5 we show the scaling of the field enhancement $\kappa$ as a function of distance from the center of the structure (panel III-a of Fig. 1) for different values of structural disorder and dynamical noise. In panel a) the field intensity as a function of distance is shown for different values of dynamical noise. We notice that as dynamical noise increases, the

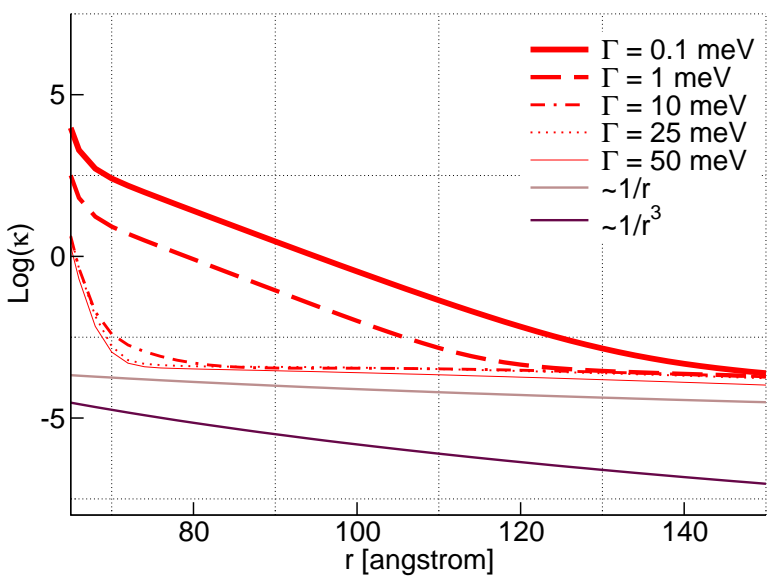

a)

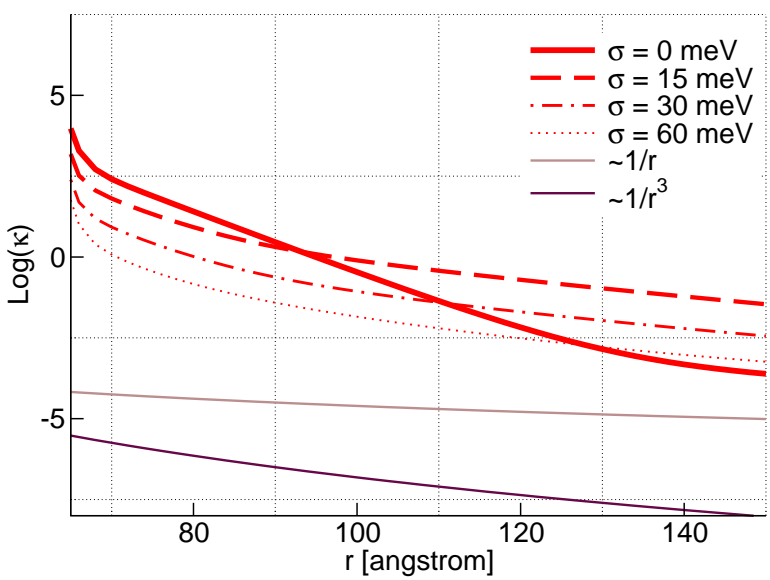

b)

Figure 5: Scaling of field enhancement $\kappa$ with dynamical noise, (panel a)) and structural disorder (panel b)) for the roll-A structure (Fig. 1; III-a) as a function of distance $r$ from the center of the structure $\left(r_{\text {center }}=0 \AA\right)$. The incoming field is polarized along the $z^{\prime}$ direction. 
field scaling tends to go as $\sim \frac{1}{r}$, but there are different slopes of the scaling on shorter distances. In panel $b$ ), the scaling as a function of structural disorder $\sigma$ is investigated at fixed small $\Gamma$. In this case the trend of the field is $\sim \frac{1}{r^{3}}$. It is not immediately clear to us why a different scaling with respect to $r$ is observed in the case of dynamical versus structural disorder, however it is interesting that perhaps nature may tune one or the other type of disorder to modify the intensity of energy transferred through structural variations. The distance amongst substructures has experimentally ${ }^{2}$ been determined to be $r_{\text {phys }} \sim 2 \mathrm{~nm}$. The slope of the fields around this distance is not homogeneous as a function of $\Gamma$. In the case of structural disorder, at about $r_{\text {phys }}$, we notice (Fig. 5, panel b) that a similar slope is observed with a smaller variation in intensity respect to panel a).

In Fig. 6, we plot the field enhancement $\kappa$ at the biological distance $r_{\text {phys }}$ for the two cylindrical structures (panels III-a and III-b of Fig. 1) as a function of dynamical noise (panel a) and structural disorder (panel b). It appears that dynamical noise has the strongest effect. In such large structures it is more likely that the main source of disorder is actually structural disorder however. This means that overall the excitation energy transfer is quite robust to disorder. Further, for all values of $\Gamma$ and $\sigma$, the enhancement is larger for the wild type structure. This suggest that the dipole arrangement of the wild type structure leads to higher efficiency in terms of field enhancement. This is also observed at the physiological distance, $r_{\text {phys }}$, relevant for transport.

\section{Energy flux}

The main role of the chlorosome is that of transmitting the collected solar energy: it is therefore important to investigate how fast energy is transferred amongst these nanostructures. In our electrodynamic model, the energy flow can be obtained from the induced fields and polarizations. In particular, one defines some acceptor molecules which are not initially excited by the incoming field and some donor molecules which are excited by the incoming field and later interact with the acceptors.

The rates of energy flow absorbed by the accep- tors, $R(t)$, can be obtained from the divergence of the pointing vector as $R(t)=\sum_{\text {acc }} \vec{E}_{\text {acc }}(t)$. $\frac{d}{d t} \vec{p}_{\text {acc }}(t)$, where $\vec{E}_{\text {acc }}$ is the electric field at the acceptor at time $t$ and $\vec{p}_{\text {acc }}$ is the induced polarization of the acceptor at time $t$. This approach has been discussed in Ref. 22.

In Fig. 7, panel a), we show these fluxes of energy as a function of time, $R(t)$, for transfer from a roll of donors (structure III-a of Fig. 1) to a dipole acceptor positioned at different distances from the center of the cylinder. The dipole acceptor is oriented vertically along the main axis of the cylinder and has transition frequency $\omega_{\text {acc }}=1.51 \mathrm{eV}$, in the region where the roll absorbs (see Fig. 2). The external field which interacts with donors is polarized along the $z^{\prime}$ direction. The energy flux is normalized by the number of donor molecules squared, $N_{\text {donor }}^{2}$. In these calculations, the dynamic disorder rate $\Gamma=50 \mathrm{meV}$. We notice that the energy flux $R(t)$ does not decrease monotonically with distance, in fact there are some distances more favorable for transfer. This can be explained by the fact that the components of the field at each distance are not the same but may rotate. This effect can be thought of as some type of coherence between acceptor and donor.

We also computed the flux of energy transfered between two concentric cylindrical structures (Structure of Fig. 1, panel II-a) for an external field polarized along the $z^{\prime}$ direction. The resulting fluxes are shown in Fig. 7, panel b) for two different values of the dynamic rate constant $\Gamma$. Here, $R_{A}$ indicates the flux when roll-A (the roll with the smaller diameter) is the acceptor and rollB the donor and $R_{A}$ indicates the opposite case. When $\Gamma=50 \mathrm{meV}$, i.e. the incoherent limit, the fluxes from roll-A and B are equivalent, as expected and decay within $300 \mathrm{fs}$. When the disorder is decreased, the flux is much larger and decays fully only after about $800 \mathrm{fs}$. Further, in this case the fluxes are different in each direction (from rollA to B and from B to A). This model however does not account for relaxation, therefore the estimated fluxes should be considered upper bounds of the actual rates. 


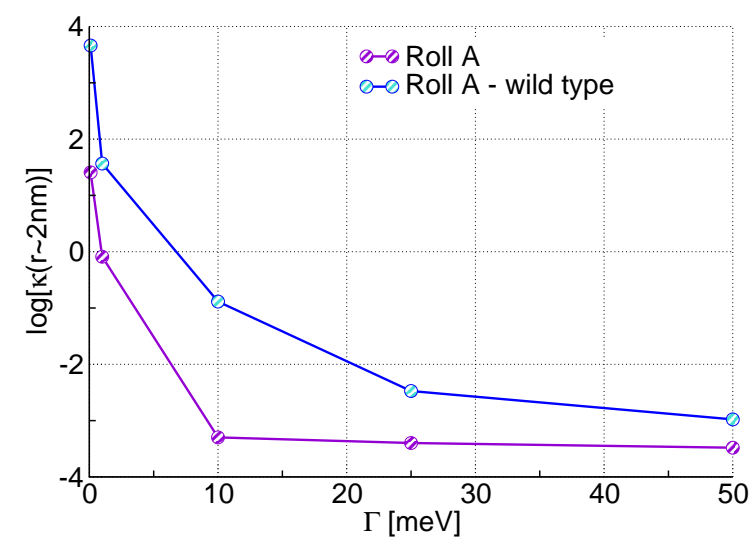

a)

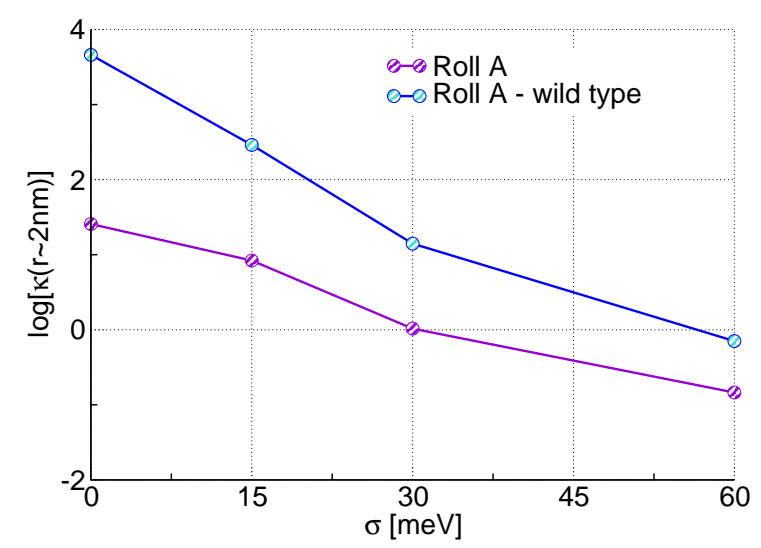

b)

Figure 6: Panel a) scaling of field enhancement $\kappa$ with dynamical noise rate $\Gamma$ for each structure, at a distance of $r_{\text {phys }}=2 \mathrm{~nm}$. Panel b) scaling of field with structural disorder $\sigma$ for each structure, at a distance of $r_{\text {phys }}=2 \mathrm{~nm}$. The incoming field is polarized along the $z^{\prime}$ direction. Roll-A and Roll-A wild type, correspond to the structures in panels III-a) and b) of Fig. 1.

\section{Conclusions}

We have analyzed the chlorosome antenna complex in the green sulfur bacteria Chlorobiumtepidum using an electrodynamic model. Three structures were considered as models for the natural chlorosome complex. Each antenna structure shows robustness to structural variations. This effect was seen in the values of the field enhancement which is much more sensitive to noise than to structural disorder.

At the physiological distance, the minimum distance observed for packing amongst nanostructures, the field enhancement trend follows different slopes as a function of disorder. Therefore no clear trend regarding variations of induced fields and polarization as a function of disorder can be deduced. However, at this same distance, the wild type nanostructure antenna shows larger field enhancement. This suggests that the molecular packing can be tuned to maximize transport properties. A preferential direction for transfer is observed consistently for all structures for specific polarizations. This suggests that this type of structure acts as a concentrator by enhancing transport for specific photon polarizations. This is also confirmed by the patterns of field depolarization which strongly depend on initial field polarization but not on structural variations. In particular we observe that the field is concentrated in the radial direction and at the edges of the cylinders (depending of the incoming field polarization), which would enable exciton transport to neighboring structures and to other layers. The field depolarization also supports transport to neighboring layers. Finally, transport has been quantified by calculating the flux of electromagnetic energy transferred between the cylindrical structure and a dipole acceptor. In this case there is a specific distance which maximizes transport. One can think of it in terms of coherence amongst the donor field polarization and the acceptor molecule dipole orientation. The timescale for the flux of energy transfer is roughly $300 \mathrm{fs}$ which is an upper bound to the true timescale, in fact the model does not account for relaxation. We also computed these fluxes of energy for transfer amongst cylindrical structures and found that depending on dynamic noise, it is enhanced in the radial direction. This study opens the road to the possibility of creating antennae that mimic this type of natural system. For instance, we could consider the idea of the chlorosome outside of its natural environment: if one could devise a nanoantenna based on the structural arrangement of dipoles in the chlorosome, how efficient would it be? 


\section{Methods}

\section{Physical model of the chlorosome struc- ture}

In this section, we describe the structures employed as models of the chlorosome in more detail. In both of the molecular packing motifs employed to construct the chlorosome structures, BChl molecules are stacked in columns such that the oxygen from the hydroxyl, $\mathrm{OH}$, group of one molecule, see Fig. 1 panel II-b), binds to the $\mathrm{Mg}$ atom of the next molecule. ${ }^{37}$ The columns of $\mathrm{BChl}$ molecules couple to each other through $\mathrm{OH} \cdots \mathrm{O}$ hydrogen bonds thus forming two dimensional layers (this is shown in Fig. 8). These layers are then folded to form cylinders or curved lamellar structures. ${ }^{33}$ Two distinct types of layer folding have been proposed previously. In Ref. 2

a)
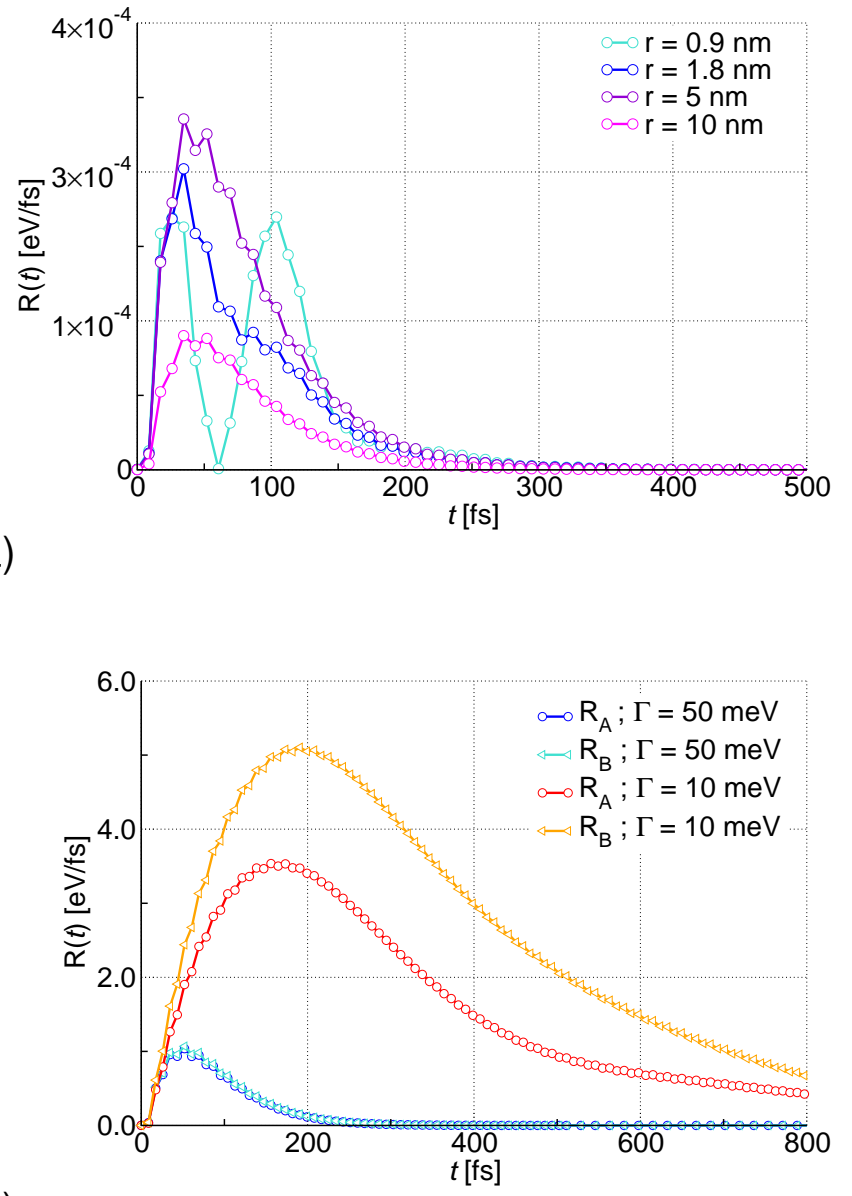

b)

Figure 7: Panel a) Flux of energy transferred from roll-A (Fig. 1, III-a) to a single dipole acceptor, oriented along the $z$ direction and located at various distances $r$ from the outside of the roll along the radial direction, here $\Gamma=50 \mathrm{meV}$. Panel $\mathrm{b}$ ) Flux of energy transferred from roll-A to roll-B, $R_{B}(t)$ and from roll-B to roll- $\mathrm{A}, R_{A}(t)$ for different values of the dynamic noise constant $\Gamma$. The incoming field is polarized along the $z^{\prime}$ direction. the authors suggested that BChl layers are folded such that BChl columns form concentric rings. This structure was obtained by fitting the $2 \mathrm{D} \mathrm{nu-}$ clear magnetic resonance (NMR) spectra of mutant Chlorobium tepidum bacteria which produce chlorosomes with BChl-d molecules. This packing motif was also supported by cryo-electron microscopy images. ${ }^{30}$ The first structure we consider (III-a in Fig. 1) comes from these studies. In contrast, in Ref. 38 the same group of authors used a different folding pattern, where BChl columns are parallel to the cylinder's symmetry axis. The latter structure was also supported by 2D NMR studies of a different mutant bacteria, which is more similar to wild-type species (the chlorosome was packed with BChl-c). The choice of these cylindrical structures is also bolstered by recent $2 \mathrm{D}$ fluorescence polarization microscopy experiments. ${ }^{36}$

We used this second type of folding to obtain the second structure, i.e III-b in Fig. 1. Finally, the layer structures are packed inside of an ellipsoidal shaped body: the chlorosome. While the chlorosome may contain multiple rolls packed in parallel, ${ }^{39}$ here we use a different model (structure III-c in in Fig. 1) which is composed of concentric rolls. ${ }^{33}$ This concentric assembly is in-line with the cryo-EM images. ${ }^{30}$ More details on this structure are given in the Supporting information.

In the electromagnetic model, each molecule is represented by a transition dipole, in particular we 


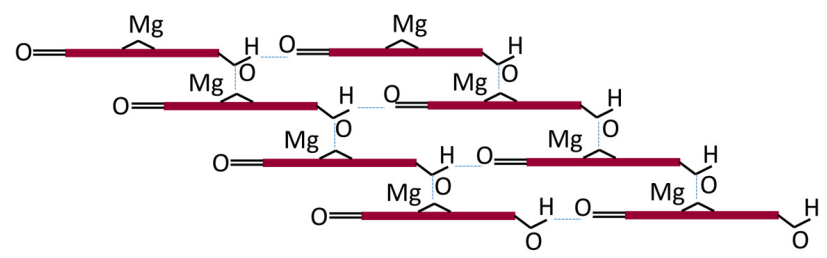

Figure 8: Two columns of BChl molecules linked together through a network of hydrogen bonds.

use the $Q_{y}$ transition dipole. The frequency of the transition $^{40}$ is taken to be $\omega_{0}=1.904 \mathrm{eV}$ for BChl$\mathrm{d}$ and $\omega_{0}=1.881 \mathrm{eV}$ for BChl-c as obtained experimentally. The dipole intensity is taken to be $\mu=5.48 \mathrm{D}$, following the values given in literature, e.g. Ref. 41, 42, 39.

\section{Polarizability model}

The system considered is an array of aggregated BChl monomers (as shown in Fig. 1). In quantum models, a Hamiltonian in the molecule localized basis $|n\rangle$ is often used to describe this type of system

$\hat{H}_{0}=\sum_{n} \epsilon_{n}|n\rangle\langle n|+\sum_{n<m} J_{n m}(|n\rangle\langle m|+| m\rangle\langle n|)$.

The energy $\epsilon_{n}$ of the $n-t h$ monomer is typically taken to be the first excited state energy, and all higher excited states are ignored. Such approximation is valid so long as the higher energy states of the monomers are well separated from the first. This energy corresponds to the $Q_{y}$ transition for BChl's. $J_{m n}$ indicates the coupling between monomers and $|n\rangle$ is the localized basis in which the $n-t h$ molecule is excited. When the molecules interact with radiation, an extra interaction term with the field must be added to this expression $V_{\text {field }}$, so that the overall Hamiltonian can be divided into a time independent Hamiltonian $\hat{H}_{0}$ and a time dependent interaction term $V_{\text {field }}$. $\hat{H}(t)=\hat{H}_{0}+V_{\text {field }}(t)$. One can then write an equation of motion for the system density matrix as a function of the Hamiltonian and solve it perturbatively in orders of interaction with the field. From the density matrix equation optical properties can be obtained (Sec. Optical properties).

A similar approach can be followed using molecular polarizabilities. Each molecular tran- sition $s$ is approximately described by an electronic transition dipole $\vec{\mu}_{n, s}$ (for the $n-t h$ molecule), ${ }^{34,43,44}$ and the frequency of the transition is taken to be $\omega_{n, s}=\epsilon_{n, s} / \hbar$. The expression for the overall electric field at the $n-t h$ molecule located at $\vec{x}_{n}$ is the sum of the internal field $\vec{E}_{\text {int }}$ which comes from the interactions with all other dipoles and the external field $\vec{E}_{\text {ext }}$,

$$
\vec{E}\left(t, \vec{x}_{n}\right)=\vec{E}_{\text {int }}\left(t, \vec{x}_{n}\right)+\vec{E}_{\text {ext }}\left(t, \vec{x}_{n}\right) .
$$

In particular, in the case of classical dipoles this equation can be written as ${ }^{34}$

$\vec{E}\left(t, \vec{x}_{n}\right)=4 \pi k^{2} \sum_{m \neq n} \mathbf{G}\left(\vec{x}_{m}, \vec{x}_{n}\right) \cdot \vec{p}_{n}+\vec{E}_{\text {ext }}\left(t, \vec{x}_{n}\right)$.

Here, $\vec{p}_{n}$ is the induced electric dipole moment at molecule $n$ due to the electric field and the constant $k$ is the magnitude of the wavevector of the incident light. The free-space Green's function tensor $\mathbf{G}$ includes all interactions amongst dipoles and is directly proportional to the field at point $\vec{x}$ due to a dipole oscillating at position $\vec{x}_{n}$. It is expressed as

$$
\begin{aligned}
\mathbf{G}\left(\vec{x}_{m}, \vec{x}_{n}\right) & =g(r)\left[(\mathbf{1}-\vec{e} \vec{e})-\left(\frac{1-i k r}{k^{2} r^{2}}\right)(\mathbf{1}-3 \vec{e} \vec{e})\right] \\
& -\frac{\mathbf{1}}{3 \epsilon_{0} k^{2}} \delta^{3}(\vec{r}),
\end{aligned}
$$

with $g(r)=\exp (i k r) /\left(4 \pi \epsilon_{0} r\right)$. In Eq. 5 the last term compensates for self-interaction. The unit vector $\vec{e}$ is defined as $\vec{e} \equiv \frac{\vec{r}}{r}$ with $\vec{r}=\vec{x}_{m}-\vec{x}_{n}$ and $r=|\vec{r}|$. The field at each molecule and the polarization can be obtained by solving the linear system of equations (Eq. 4) with $n=1, \ldots, N$ where $N$ is the number of molecules in the aggregate. Details of how these equations can be solved efficiently for large systems can be found in Ref. 23. The field enhancements and depolarizations as presented in the "Results" section, are obtained by solving for the induced dipole moments of all the molecules (Eq. 4). 


\section{Optical properties}

\section{Linear absorption}

Using the formalism introduced in "Polarizability model" section, an equation for the induced electric dipole $\vec{p}$ on each molecule can be obtained as a function of the index of refraction. ${ }^{34,43}$ In the limit of the dipole approximation, the expression for the molar extinction coefficient is then

$$
\epsilon_{\mathrm{abs}}=-\frac{4 \pi \omega}{3000 \cdot \ln (10) c} \mathcal{N}_{\mathrm{av}} \sum_{i j} \operatorname{Im} \mathbf{A}_{i j} \vec{u}_{i} \cdot \vec{u}_{j},
$$

with $\vec{u}_{i}=\frac{\vec{\mu}_{i}}{\left|\overrightarrow{\mu_{i}}\right|}$. Here the matrix $\mathbf{A}$ is defined as

$$
\mathbf{A}_{i j}=\left[\frac{\delta_{i j}}{\chi_{i}}+\mathbf{D}_{i j}\right]^{-1}
$$

with $\mathbf{D}_{i j}=\vec{u}_{i} \cdot \mathbf{G}_{i j} \cdot \vec{u}_{j}$, the term which includes dipole-dipole interactions and $\chi_{i}$ the molecular response function as defined in Eq. 1. In the limit of static dipole interactions, the tensor $\mathbf{G}$ takes the simple form $\mathbf{G}_{i j} \equiv \mathbf{G}\left(\overrightarrow{x_{i}}, \vec{x}_{j}\right)=\frac{3 \vec{e} e-1}{r^{3}}$.

To better understand where resonances occur, we can look at the eigenvalues of $\mathbf{A}$. For zero dynamical noise rate $\Gamma$ and for identical molecules $\left(\vec{\mu}_{k} \equiv \vec{\mu}, \omega_{k}=\omega_{\text {mol }}\right)$ we see that resonances are the roots of

$$
\Omega_{\mathrm{agg}, k}^{2} \equiv \omega_{\mathrm{mol}}^{2}+2 \frac{|\tilde{\mu}|^{2}}{\hbar} \gamma_{k} \omega_{\mathrm{mol}} .
$$

Here, $\gamma_{k}$ is the $k-t h$ eigenvalue of G. We can define $\frac{|\vec{\mu}|^{2}}{\hbar} \gamma_{k}=\tilde{\gamma}_{k}$ to get proper units of energy for the coupling. Thus $\Omega_{\mathrm{agg}, k}^{2} \equiv \omega_{\mathrm{mol}}^{2}+2 \tilde{\gamma}_{k} \omega_{\mathrm{mol}}$. In this expression it is clear that the aggregate resonances will be shifted respect to the molecular transition frequency $\omega_{\mathrm{mol}}$ and this shift will depend on the coupling between monomers, here captured by $\gamma_{k}$. Eq. 6 was employed to compute the linear absorption spectrum shown in Fig. 2. A similar approach, the CES method, has also been used for excitonic systems ${ }^{14}$ and leads essentially to the same equations that are used in the present work.

Now, in the quantum case, the simplest approach to obtain the absorption spectrum consists in applying Fermi's Golden rule

$$
\epsilon_{\mathrm{abs}}^{(\mathrm{qtm})}(\omega)=\frac{4 \pi \omega}{3 c} \sum_{k}|\tilde{\mu}|^{2} \pi \delta\left(E_{k}-\hbar \omega\right),
$$

here, $E_{k}$ is the $k-t h$ eigenvalue of the Hamiltonian given in Eq. 2 and $\vec{\mu}$ is it's transition dipole. In this case resonances in the aggregate spectrum will be obtained as the roots of $\Omega_{\mathrm{agg}, \mathrm{k}}^{2}=E_{k}^{2} / \hbar^{2}=$ $\left(\omega_{\mathrm{mol}}+\tilde{\gamma}_{k}\right)^{2}$. The expression given in Eq. 9 is the one used to compute the quantum absorption spectrum shown in Fig. 2. We see that respect to the classical case (Eq. 8), the frequencies squared are shifted by $\tilde{\gamma}_{k}^{2}$ : this comes from the absence of counter rotating terms in the classical response function. ${ }^{31}$ In fact, in classical electromagnetics only one type of time ordering of interactions between photons and molecules is allowed. For optical frequencies the counter-rotating term only gives a small shift, however it becomes important for long wavelengths, for instance, in microwave cavities. It should be noted that the counterrotating term is still partly included in our model through the susceptibility functions of the single molecules. However, its contribution to the intermolecular interaction is not accounted for. Another way to see how the shift arises is by comparison of the eigenstates of a quantum Hamiltonian with the normal modes for a set of driven coupled classical oscillators. The comparison can be made after applying the Dirac mapping and realistic coupling (RCA) approximation. This approach has been discussed in Ref. 45. The two approaches become equivalent in the limit where the couplings are small compared to the monomer transition frequency $\gamma_{k} \lll \omega_{\text {mol }}$.

\section{Circular dichroism}

Circular dichroism is another important optical quantity which can help identify the correct structure of a system. It can also be obtained both classically and quantum mechanically. Following the classical approach ${ }^{34}$ of the previous sections, one can arrive at the following expression for the molar ellipticity

$$
\theta=-\sum_{i j} \mathbf{C}_{i j} \operatorname{Im} \mathbf{A}_{i j} .
$$

Here we have used the standard definition of CD, as the difference between left polarized and right 
polarized intensity. The matrix $\mathbf{C}$ is defined as

$$
\mathbf{C}_{i j}=\frac{6 \omega^{2} \mathcal{N}_{\mathrm{av}}}{c^{2}}\left(\vec{u}_{i} \times \vec{u}_{j}\right) \cdot\left(\vec{x}_{i}-\vec{x}_{j}\right)
$$

in the absence of any magnetic dipoles or polarizations. Here, $\vec{x}_{i}$ corresponds to the position of the $i-t h$ molecule respect to the origin.

Similarly, in the quantum case one finds (see e.g. Ref. 46) that in the exciton basis, i.e. the basis in which the Hamiltonian of Eq. 2 is diagonal, the rotation strength associated with the exciton level $J$ is

$$
R_{J}=-\frac{6 \omega^{2} \mathcal{N}_{\mathrm{av}}}{c^{2}} \sum_{i k} \sigma_{J i} \sigma_{J k}\left(\vec{u}_{i} \times \vec{u}_{j}\right) \cdot\left(\vec{x}_{i}-\vec{x}_{j}\right) .
$$

The coefficients $\sigma$ are the coefficients of the matrix $\mathbf{S}$ which diagonalizes the Hamiltonian of the system. Eq. 10 and eq. 12 are used in this work to compute the circular dichroism spectra shown in Fig. 2.

Supporting Information Available: A figure and detailed description of the third model structure for the chlorosome are included in the supporting information. This material is available free of charge via the Internet at http://pubs.acs.org.

\section{Acknowledgments}

The authors would like to thank A. Eisfeld for useful discussions. S. V. acknowledges support from the Center for Excitonics, an Energy Frontier Research Center funded by the U.S. Department of Energy, Office of Science and Office of Basic Energy Sciences under Award Number DESC0001088 as well as support from the Defense Advanced Research Projects Agency under award number N66001-10-1-4060. S.K.S. and A.A.-G. acknowledge Defense Threat Reduction Agency grant HDTRA1-10-1-0046. D. A.-O.-B., M. R. and H. M. are grateful to the U.S. Air Force Office of Scientific Research (AFOSR) Grant No. FA9550-10-1-0438, for the support of this work.

\section{References}

1. Blankenship, R. E. Molecular Mechanisms of Photosynthesis; World Scientific: London, 2002.

2. Ganapathy, S.; Oostergetel, G. T.; Wawrzyniak, P. K.; Reus, M.; Gomez Maqueo Chew, A.; Buda, F.; Boekema, E. J.; Bryant, D. A.; Holzwarth, A. R.; de Groot, H. J. M. Alternating Syn-Anti Bacteriochlorophylls Form Concentric Helical Nanotubes in Chlorosomes. Proc. Natl. Acad. Sci. 2009, 106, 8525-8530.

3. Devilez, A.; Stout, B.; Bonod, N. Compact Metallo-Dielectric Optical Antenna for Ultra Directional and Enhanced Radiative Emission. ACS nano 2010, 4, 3390-3396.

4. King, N. S.; Knight, M. W.; Large, N.; Goodman, A. M.; Nordlander, P.; Halas, N. J. Orienting Nanoantennas in Three Dimensions To Control Light Scattering Across a Dielectric interface. Nano Lett. 2013, 13, 5997-6001.

5. Novotny, L.; van Hulst, N. Antennas for Light. Nat. Photonics 2011, 5, 83-90.

6. Knight, M. W.; Sobhani, H.; Nordlander, P.; Halas, N. J. Photodetection with Active Optical Antennas. Science 2011, 332, 702-704.

7. Knight, M. W.; Liu, L.; Wang, Y.; Brown, L.; Mukherjee, S.; King, N. S.; Everitt, H. O.; Nordlander, P.; Halas, N. J. Aluminum Plasmonic Nanoantennas. Nano Lett. 2012, 12, 6000-6004.

8. Schuller, J.; Barnard, S. E.; Cai, W.; Jun, Y. C.; White, J. S.; Brongersma, M. L. Plasmonics for Extreme Light Concentration and Manipulation. Nat. Mater. 2010, 9, 193 204.

9. Hernández-Martínez, P. L.; Govorov, A. O.; Demir, H. V. Generalized Theory of FörsterType Nonradiative Energy Transfer in Nanostructures with Mixed Dimensionality. J. Phys. Chem. C 2013, 117, 10203-10212. 
10. Fujita, T.; Brookes, J. C.; Saikin, S. K.; Aspuru-Guzik, A. Memory-Assisted Exciton Diffusion in the Chlorosome LightHarvesting Antenna of Green Sulfur Bacteria. J. Phys. Chem. Lett. 2012, 3, 2357-2361.

11. Fujita, T.; Huh, J.; Saikin, S. K.; Brookes, J. C.; Aspuru-Guzik, A. Theoretical Characterization of Excitation Energy Transfer in Chlorosome Light-Harvesting Antennae from Green Sulfur Bacteria. Photosynth. Res., in press 2014,

12. Huh, J.; Saikin, S. K.; Brookes, J.; Valleau, S.; Fujita, T.; Aspuru-Guzik, A. Atomistic Study of Energy Funneling in the Light-Harvesting Complex of Green Sulfur Bacteria. J. Am. Chem. Soc. 2014, 136, 2048-2057.

13. Prokhorenko, V. I.; Steensgaard, D. B.; Holzwarth, A. R. Exciton Theory for Supramolecular Chlorosomal Aggregates: 1. Aggregate Size Dependence of the Linear Spectra. Biophys. J. 2003, 85, 3173-3186.

14. Eisfeld, A.; Kniprath, R.; Briggs, J. S. Theory of the Absorption and Circular Dichroism Spectra of Helical Molecular Aggregates. J. Chem. Phys. 2007, 126, 104904(1)104904(13).

15. Beatty, J. T.; Overmann, J.; Lince, M. T.; Manske, A. K.; Lang, A. S.; Blankenship, R. E.; Van Dover, C. L.; Martinson, T. A.; Plumley, F. G. An Obligately Photosynthetic Bacterial Anaerobe from a DeepSea Hydrothermal Vent. Proc. Natl. Acad. Sci. 2005, 102, 9306-9310.

16. Overmann, J.; Cypionka, H.; Pfennig, N. An Extremely Low-Light-Adapted Phototrophic Sulfur Bacterium from the Black Sea. Limnol. Oceanog. 1992, 37, 150-155.

17. Ishizaki, A.; Fleming, G. R. Theoretical Examination of Quantum Coherence in a Photosynthetic System at Physiological Temperature. Proc. Natl. Acad. Sci. 2009, 106, 1725517260.

18. Pachón, L. A.; Brumer, P. Computational Methodologies and Physical insights into
Electronic Energy Transfer in Photosynthetic Light-Harvesting Complexes. Phys. Chem. Chem. Phys. 2012, 14, 10094-10108.

19. Ritschel, G.; Roden, J.; Strunz, W. T.; AspuruGuzik, A.; Eisfeld, A. Absence of Quantum Oscillations and Dependence on Site Energies in Electronic Excitation Transfer In the Fenna-Matthews-Olson Trimer. J. Phys. Chem. Lett. 2011, 2, 2912-2917.

20. Shim, S.; Rebentrost, P.; Valleau, S.; AspuruGuzik, A. Atomistic Study of the Long-Lived Quantum Coherences in the Fenna-MatthewsOlson Complex. Biophys. J. 2012, 102, 649660.

21. Valleau, S.; Eisfeld, A.; Aspuru-Guzik, A. On the Alternatives for Bath Correlators and Spectral Densities from Mixed QuantumClassical Simulations. J. Chem. Phys. 2012, 137, 224103(1)-224103(13).

22. Zimanyi, E. N.; Silbey, R. J. Unified Treatment of Coherent and incoherent Electronic Energy Transfer Dynamics using Classical Electrodynamics. J. Chem. Phys. 2010, 133, 144107(1)-144107(10).

23. Ansari-Oghol-Beig, D.; Rostami, M.; Chernobrovkina, E.; Saikin, S.; Valleau, S.; Mosallaei, H.; Aspuru-Guzik., A. Parametric Hierarchical Matrix Approach for the Wideband Optical Response of Large-Scale Molecular Aggregates. J. App. Phys. 2013, 114, 164315(1)164315(10).

24. van Rossum, B.-J.; Steensgaard, D. B.; Mulder, B. G. J., F. M. and; Schaffner, K.; Holzwarth, A. R.; de Groot, H. J. M. Refined Model of the Chlorosomal Antennae of the Green Bacterium Chlorobium Tepidum from Proton Chemical Shift Constraints Obtained With High-Field 2d and 2-D Mas Nmr Dipolar Correlation Spectroscopy. Biochem. 2001, 40, 1587-1595.

25. de Boer, I.; Matysik, J.; Erkelens, K.; Sasaki, S.; Miyatake, T.; Yagai, S.; Tamiaki, H.; Holzwarth, A. R.; de Groot, H. J. M. Mas Nmr Structures of Aggregated 
Cadmium Chlorins Reveal Molecular Control of Self-Assembly of Chlorosomal Bacteriochlorophylls. J. Phys. Chem. B 2004, 108, 16556-16566.

26. Psencík, J.; Ikonen, T. P.; Laurinmäki, P.; Merckel, M. C.; Butcher, S. J.; Serimaa, R. E.; Tuma, R. Lamellar Organization of Pigments in Chlorosomes, the Light Harvesting Complexes of Green Photosynthetic Bacteria. Biophys. J. 2004, 87, 1165-1172.

27. Hohmann-Marriott, M. F.; Blankenship, R. E.; Roberson, R. W. The Ultra Structure of Chlorobium Tepidum Chlorosomes Revealed By Electron Microscopy. Photosynth. Res. 2005, 86, 145-154.

28. Egawa, A.; Fujiwara, T.; Mizoguchi, T.; Kakitani, Y.; Koyama, Y.; Akutsu, H. Structure of the Light-Harvesting Bacteriochlorophyll $\mathrm{C}$ Assembly in Chlorosomes from Chlorobium Limicola Determined By Solid-State Nmr. Proc. Natl. Acad. Sci. 2007, 104, 790-805.

29. Linnanto, J. M.; Korppi-Tommola, J. E. I. Investigation on Chlorosomal Antenna Geometries: Tube, Lamella and Spiral-Type SelfAggregates. Photosynth. Res. 2008, 96, 227245.

30. Oostergetel, G. T.; Reus, M.; Gomez Maqueo Chew, A.; Bryant, D. A.; Boekema, E. J.; Holzwarth, A. R. Long-Range Organization of Bacteriochlorophyll in Chlorosomes of Chlorobium Tepidum Investigated By CryoElectron Microscopy. FEBS lett. 2007, 581, 5435-9.

31. Mahan, G. D. Davydov Splittings in Anthracene. J. Chem. Phys. 1964, 41, 29302933.

32. Martiskainen, J.; Linnanto, J.; Aumanen, V.; Myllyperkiö, P.; Korppi-Tommola, J. Excitation Energy Transfer in Isolated Chlorosomes from Chlorobaculum tepidum and Prosthecochloris aestuarii. Photochem. Photobiol. 2012, 88, 675-683.

33. Tang, J. K. H.; Saikin, S. K.; Pingali, S. V.; Enriquez, M. M.; Huh, J.; Frank, H. A.;
Urban, V. S.; Aspuru-Guzik, A. Temperature and Carbon Assimilation Regulate the Chlorosome Biogenesis in Green Sulfur Bacteria. Biophys. J. 2013, 105, 1346-1356.

34. DeVoe, H. Optical Properties of Molecular Aggregates. II Classical Theory of the Refraction, Absorption, and Optical Activity of Solutions and Crystals. J. Chem. Phys. 1965, 43, 3199-3208.

35. Shibata, Y.; Saga, Y.; Tamiaki, H.; Itoh, S. Polarized Fluorescence of Aggregated Bacteriochlorophyll $\mathrm{C}$ and Baseplate Bacteriochlorophyll a in Single Chlorosomes Isolated from Chloroflexus Aurantiacus. Biochemistry 2007, 46, 7062-7068.

36. Tian, Y.; Camacho, R.; Thomsson, D.; Reus, M.; Holzwarth, A. R.; Scheblykin, I. G. Organization of Bacteriochlorophylls in Individual Chlorosomes from Chlorobaculum Tepidum Studied by 2-Dimensional Polarization Fluorescence Microscopy. J. Am. Chem. Soc. 2011, 133, 17192-17199.

37. Holzwarth, A. R.; Schaffner, K. On the Structure of Bacteriochlorophyll Molecular Aggregates in the Chlorosomes of Green Bacteria. a Molecular Modelling Study. Photosynth. Res. 1994, 41, 225-233.

38. Ganapathy, S.; Oostergetel, G. T.; Reus, M.; Tsukatani, Y.; Gomez Maqueo Chew, A.; Buda, F.; Bryant, D. A.; Holzwarth, A. R.; de Groot, H. J. M. Structural Variability in Wild-Type and Bchq Bchr Mutant Chlorosomes of the Green Sulfur Bacterium Chlorobaculum Tepidum. Biochemistry 2012, $51,4488-4498$.

39. Prokhorenko, V. I.; Steensgaard, D. B.; Holzwarth, A. R. Exciton Dynamics in the Chlorosomal Antennae of the Green Bacteria Chloroflexus Aurantiacus and Chlorobium Tepidum. Biophys. J. 2000, 79, 2105-2120.

40. Linnanto, J.; Korppi-tommola, J. Spectroscopic Properties of $\mathrm{Mg}$-Chlorin , $\mathrm{Mg}$ Bacteriochlorin, and Bacteriochlorophylls a , 
b , c , d , e , f, g , and h Studied by Semiempirical and Ab initio MO / CI Methods. J. Phys. Chem. 2001, 3855-3866.

41. Knox, R. S.; Spring, B. Q. Dipole Strengths in the Chlorophylls. Photochemistry and Photobiology 2003, 77, 497-501.

42. Adolphs, J.; Renger, T. How Proteins Trigger Excitation Energy Transfer in the Fmo Complex of Green Sulfur Bacteria. Biophys. J. 2006, 91, 2778-2797.

43. Keller, D.; Bustamante, C. Theory of the interaction of Light With Large Inhomogeneous Molecular Aggregates. I. Absorption. $J$. Chem. Phys. 1986, 84, 2961-2971.

44. Keller, D.; Bustamante, C. Theory of the interaction of Light With Large Inhomogeneous Molecular Aggregates. Ii. Psitype Circular Dichroism. J. Chem. Phys. 1986, 84, 2972-2980.

45. Briggs, J. S.; Eisfeld, A. Coherent Quantum States from Classical Oscillator Amplitudes. Phys. Rev. A 2012, 85, 052111(1)052111(10).

46. Somsen, O. J. G.; van Grondelle, R.; van Amerongen, H. Spectral Broadening of interacting Pigments: Polarized Absorption By Photosynthetic Proteins. Biophys. J. 1996, 71, 1934-1951. 